\title{
BIO_08 - Development of anti-SARS-CoV-2 specific scFv antibody library from convalescent plasma of COVID-19 recovered patients using phage display technology
}

Kafil Ahmed ${ }^{1 *}$; Vyankatesh Pidiyar ${ }^{1}$; Syed Ahmed ${ }^{1}$; Sanket Shah ${ }^{1}$.

${ }^{1}$ Techinvention Lifecare Pvt Ltd.

Introduction: The ongoing pandemic of COVID-19 has spread nearly to every country in less than 6 months and most of these countries are already facing second wave of infection. Due to rapid mutational capacity, the new variants of SARS-CoV-2 have been reported in different countries which claimed more than 103 million cases globally. According to WHO guidelines, the current treatments are only supportive and there is need for development of novel antivirals to control the spread of mutant viruses. Development of antibody targeting specific protein is the most crucial way to combat the infection. Despite being largest and fastest growing sector, production of monoclonal antibody $(\mathrm{mAb})$ based therapeutics has limitations of time consuming and costly manufacturing process. To overcome this, the development of single-chain variable fragments ( $\mathrm{scFv}$ ) antibodies can be attractive alternative to mAbs. The convalescent plasma from the COVID-19 recovered patients can be rich source for isolation of anti-SARS-CoV-2 antibody genes for $\mathrm{scFv}$ production. We have generated two $\mathrm{scFv}$ libraries using phage display technology from convalescent plasma of 10 COVID-19 recovered patients. The library now with us includes one billion different clones of antibody genes (VHs-Vks and VHs-VLs) and has high probability to get high affinity fully human antibodies. These libraries were panned against three target proteins S1, S2 and Receptor Binding Domain (RBD) of SARS-CoV-2 and 1500 clones are being screened for isolation of neutralizing scFv against SARS-CoV-2. The isolated clones can be used individually or as cocktail for antiviral therapy.

Objective: To develop anti-SARS-CoV-2 specific scFv antibody library from convalescent plasma of COVID-19 recovered patients using phage display technology.

Methodology: The convalescent plasma was collected from ten COVID-19 recovered patients. The lymphocytes were separated by Ficoll separation and used for isolation of total RNA. The antibody specific genes $(\mathrm{K} \& \mathrm{~L})$ were PCR amplified and cloned in phage display expression vector and transformed in E. coli XL1 Blue strain. The bacteriophage M13K07 was used for expression of phagemid vector. The libraries were panned against SARS-CoV-2 proteins viz: S1, S2 \& RBD.

Results: After completing bio panning process, 1500 clones were isolated for each target antigens. As a result of ELISA screening, about 3\% positive clones were obtained which are being further characterized. The best binders will be tested for virus neutralization assay, toxicological studies, preclinical and clinical studies.

Conclusion: Anti-SARS-CoV-2 specific scFc antibody library was successfully constructed from convalescent plasma of COVID-19 recovered patients with 3\% ELISA positive binders. This library will be used for the development of monoclonal antibody against SARS-CoV-2 for the developing countries. The libraries can also be used for diagnostic purposes, bioassays kit development, affinity maturation, studying protein-protein interactions and developing antibodies against other infectious diseases such as chikungunya, dengue and RSV.

Keywords: SARS-CoV-2; Antibody; Phage 